Original Research Paper

\title{
3D Printing of Porous Scaffolds for Medical Applications
}

\author{
Ablah Aljohani and Salil Desai \\ Department of Industrial and Systems Engineering, \\ North Carolina A\&T State University, Greensboro, USA
}

\section{Article history}

Received: 01-6-2018

Revised: 05-6-2018

Accepted: 13-6-2018

Corresponding Author:

Salil Desai

Department of Industrial and

Systems Engineering, North

Carolina A\&T State University,

Greensboro, USA

Email: sdesai@ncat.edu

\begin{abstract}
Custom engineered scaffolds that mimic the physiology of native tissue is an emerging need for successful regenerative medicine. One of the key challenges in current manufacturing methods is the lack of controlled porosity throughout the scaffold structure. In this research, our group explores the fabrication of tissue engineering scaffolds using 3D printing technology. The fused deposition modeling method was utilized to fabricate scaffolds with different unit-cell designs and in-fill densities. Specimens were fabricated in Acrylonitrile-Butadiene-Styrene (ABS) material for different pattern designs which include linear, hexagonal, diamond and Moroccan star. The experimental phase of this research revealed the influence unit-cell design, in-fill density and pattern orientation on the porosity of the specimens. Computational modeling using finite element analysis was conducted to study the relationship between structure, porosity and mechanical strength of the scaffolds. A comparative analysis was performed for ASTM standard specimens for tensile and compression tests for von Mises stress and displacement values. The results indicated that diamond unit-cell pattern had the highest stress and displacement. In contrast, the hexagonal honey-comb pattern had the best mechanical strength, especially for higher porosities. Specimens loaded in the compressive mode had 50\% lower stress values as compared to tensile tests. Thus, by manipulating the unit-cell type, in-fill density and pattern orientation one can fabricate a scaffold structure that balances cellular function with the load-bearing requirement for a specific application. This research lays a foundation for evaluating additive manufacturing technologies for biomedical implants by manipulating both process parameters and material properties.
\end{abstract}

Keywords: 3D Printing, Additive Manufacturing, Finite Element Analysis (FEA), Tissue Engineering, Porous Scaffolds

\section{Introduction}

Additive Manufacturing (AM) popularly called as 3D printing has influenced several medical applications (Perkins et al., 2014; Schubert et al., 2014; Perkins et al., 2015). 3D printing has been applied to tissue engineering, prosthetics fabrication, and pharmaceutical products that include drug delivery (Desai and Harrison, 2010; Desai et al., 2010; Klein et al., 2013).

$3 \mathrm{D}$ printing is the fabrication of structures layer-bylayer in multiple materials to develop functional devices (Parupelli et al., 2016). Due to its customizable nature, additive manufacturing can accelerate health care access to a wider population through medical products, drugs, and biomedical equipment (Banks, 2013). However, in spite of its significant promise, scientific and regulatory challenges remain and the technology will need time to evolve (Ursan et al., 2013; Schubert et al., 2014).

Implants and prostheses can be fabricated through the translation of x-ray, MRI, or CT scans into digital (.stl) 3D print files (Klein et al., 2013). Thus, 3D printing can be implemented to fabricate complex free-form anatomical shapes for prosthetic limbs and surgical implants. In recent years, this approach has been progressed to include the transplantation of cells along with supporting scaffolds and biomolecules for the restoration of pathologically altered tissue architectures (Liu et al., 2017). The scaffolds need to mimic the native tissue structure and provide comparative analogs of the extracellular matrix. Synthetic scaffold acts as a temporary matrix for cell proliferation wherein growth factors and nutrients can trigger cellular differentiation 
of specific tissue types. Scaffolds have been implemented in the re-engineering of bone, ligament, cartilage, skin, neural conduits, vascular tissue and skeletal muscle. Scaffold also aid as a transport system for controlled delivery of DNA, drugs, and proteins. Numerous technologies have been utilized to construct porous scaffolds to regenerate tissues in addition to the controlled release of bioactive agents for tissue engineering applications (Dhandayuthapani et al., 2011).

The level of porosity and pore size of biomaterial scaffolds are vital in bone formation (Dadsetan et al., 2008). Previous studies have investigated the relationship between porosity and pore size of the biomaterial scaffolds and their role in bone regeneration (Karageorgiou and Kaplan, 2005; Murphy et al., 2010). In the in vitro model, lower porosity stimulates osteogenesis by suppressing cell proliferation and forcing cell aggregation (Ramalingam et al., 2013). In contrast, higher porosity and pore size result in greater bone growth in vivo (Pan et al., 2015). However, an increase in the porosity can result in lower mechanical strength of the scaffold. Thereby, restricting the upper functional limit for pore size and porosity (Bencherif et al., 2013). Small pores favored hypoxic conditions and induced osteochondral formation before osteogenesis, while large pores that are well-vascularized lead to direct osteogenesis without preceding cartilage formation (Gerhardt and Boccaccini, 2010). Thus, new fabrication techniques, such as solid free form fabrication can potentially be used to produce scaffolds that can treat specific bone-repair needs (Karageorgiou and Kaplan, 2005).

Scaffolds are designed to stimulate vascularization and bone formation. These scaffolds are often porous and made of biodegradable materials that harbor different growth factors, genes, drugs, or stem cells (Luo et al., 2015). Typical fabrication methods for scaffolds generate a porosity that is uniformly distributed throughout the scaffold dimension (Hutmacher, 2001). However, this type of scaffold does not mimic natural bone which has a higher porosity in the core and a strong, dense outer shell. The porosity distribution in the scaffold can be manipulated by manufacturing methods such as $3 \mathrm{D}$ printing. This design manipulation encourages functionality and interconnectivity within the scaffolds (Bose et al., 2012).

This research aims to develop different in-fill pattern types to morph variable scaffold designs. 3D printing technique was implemented to control the porosity of the specimens for each in-fill pattern type. The mechanical strength of these scaffolds was evaluated in both tension and compression loading modes using computational modeling. Finite element analysis was conducted on ASTM specimens with different porosities. This research lays the foundation for the fabrication of custom scaffolds with controlled pattern type and density for biomedical applications.

\section{Method and Materials}

This research was composed of both experimental and computational analysis of the porous 3D printed scaffold structures. In the experimental part, the effect of different pore structures and in-fill density on the porosity of 3D printed scaffolds was evaluated. The computational section included finite element analysis of standard specimens based on ASTM compression and tension tests.

\section{Experimental Analysis of Pore Structures}

The Fused Deposition Modeling (FDM) technique was used to fabricate porous scaffolds with Acrylonitrile Butadiene styrene (ABS) polymer. Specimens with dimensions $(25.91 \mathrm{~mm} \times 25.91 \mathrm{~mm} \times 6.35 \mathrm{~mm})$ were printed with different in-fill pattern designs. The in-fill patterns represented different pore structures such as hexagonal, square, diamond and Moroccan star. The infill density for each pattern was varied from $10 \%$ to $100 \%$ in $25 \%$ increments. Figure 1 shows the 3D Printer used to print the specimens.

The weight of the specimens (Ws) was measured using a weighing scale (Mettler Toledo). The volume of the specimen (Vs) was measured using Equation 1. The porosity of the specimens was measured using Equation 2. Standard specimens as per ASTM (D63814) were fabricated with different in-fill densities in orthogonal directions.

$$
V_{s}=\frac{W s}{\rho b}
$$

\section{Where:}

$V_{s}=$ The volume of the specimen $\left(\mathrm{cm}^{3}\right)$

$W_{s}=$ The weight of the specimens $(\mathrm{g})$

$\rho b=$ The density of the bulk material (For ABS, $\rho b=$ $1.04 \mathrm{~g} / \mathrm{cm}^{3}$ )

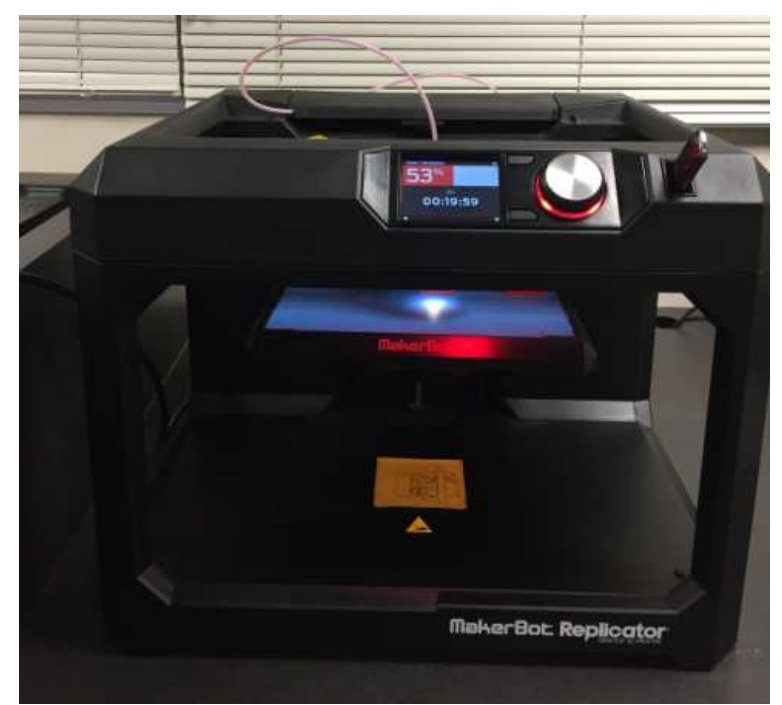

Fig. 1. MakerBot Replicator desktop 3D printer used to fabricate the specimens 


$$
p=\frac{V b-V S}{V b} \times 100
$$

Where,

$\rho=$ The porosity of the specimens

$V b=$ The volume of bulk specimen $(100 \%$ solid $)$

$V s=$ The density of the specimens

\section{Computational Modeling of 3D Printed Scaffolds}

Computational analysis was performed on the $3 \mathrm{D}$ printed scaffolds to evaluate the relationship between different pore structures and corresponding mechanical strength. In general, the relationship between the porosity and the strength of the material is an inversely proportional relationship. Thus, an increase in the porosity leads to lower mechanical strength of specimen and vice versa.
Test specimens were considered for the computational modeling based on the ASTM Tensile (D638-14) and Compressive (D695-15) standards. Figure 2 shows the detailed dimensions of the tensile and compression test specimens, respectively. Each specimen had a thickness of $3.2 \mathrm{~mm}$. Both of these specimens were modeled with different pore geometries and densities using SolidWorks CAD software. The pore geometries included unit cells of the square, circle, diamond and hexagonal structures. The pore densities for each geometry was varied at $10 \%, 25 \%, 50 \%, 75 \%$ and $100 \%$ (solid), respectively.

Finite element analysis was performed on each specimen to evaluate the deformation and von Mises stresses. A force of $100 \mathrm{~N}$ was loaded on the tensile and compression test specimens in the longitudinal direction as shown in Fig. 3.

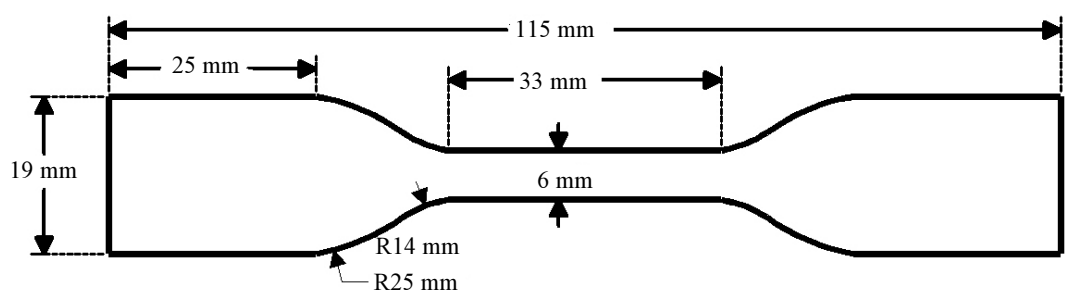

(a)

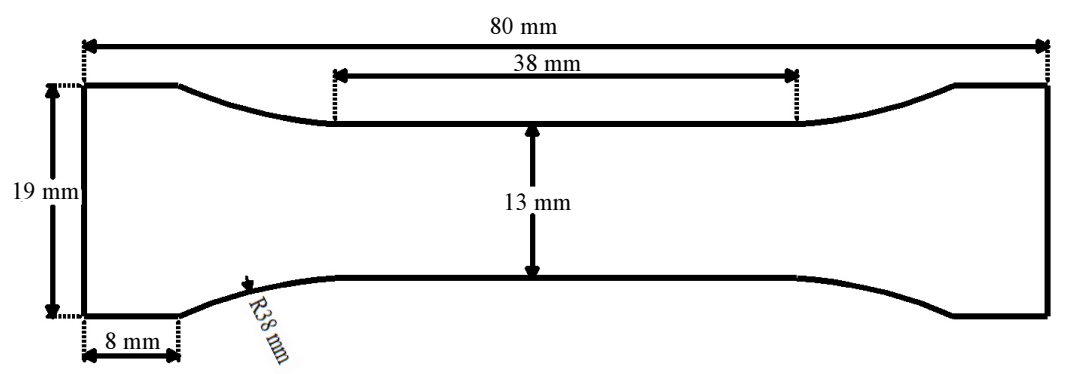

(b)

Fig. 2: ASTM specimens (a) Tensile (D638-14) and (b) Compression (D695-15)

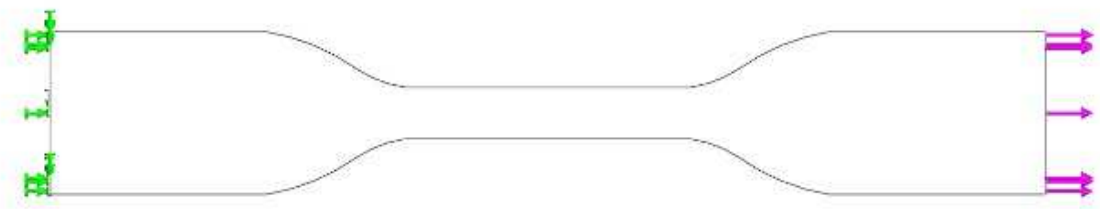

(a)

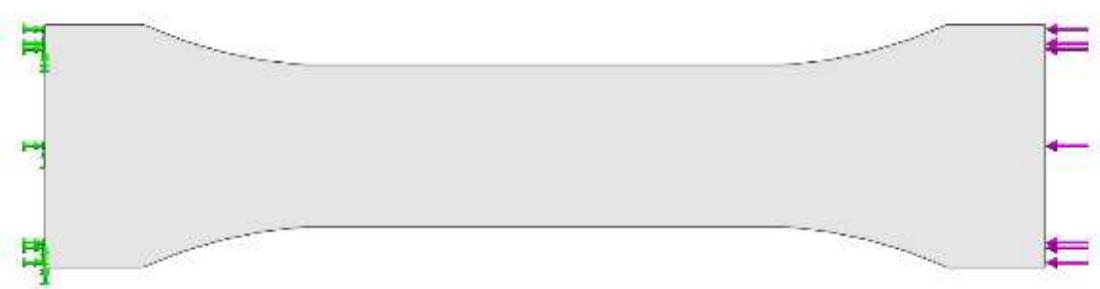

(b)

Fig. 3: ASTM specimens showing fixed boundary conditions and a force loading of $100 \mathrm{~N}$ in the longitudinal direction (a) Tensile test and (b) Compression test 


\section{Results and Discussion}

\section{Experimental Analysis of Pore Structures}

The Fused Deposition Modeling (FDM) technique was employed to fabricate porous structures for tissue engineering applications such as bone implants. Table 1 shows the specimens with different pattern designs and infill densities, respectively. As can be seen, different patterns with the same in-fill density have varying porosity. The porosity of the specimens was measured to quantify the differences based on the pattern type.
Table 2 shows the measured weights (Ws) of the specimens. Table 3 shows the volume of the specimens (Vs) calculated using Equation 1. The porosity of the specimens was measured using Equation 2. Figure 4 shows the porosity of the different pattern designs and their respective in-fill densities.

All patterns with $100 \%$ in-fill density were solid specimens ( $0 \%$ porosity). The Moroccan star pattern had lower porosity for all in-fill densities as compared to all other pattern designs. This can be attributed to a denser design pattern which led to lower porosities.

Table 1: 3D printed specimens with different pattern designs and in-fill densities

In-fill Density (\%) Linear

10

25
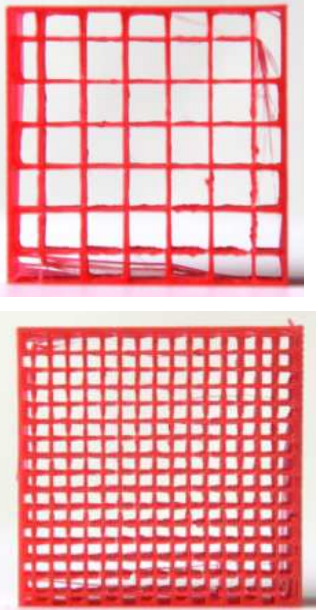

50

75
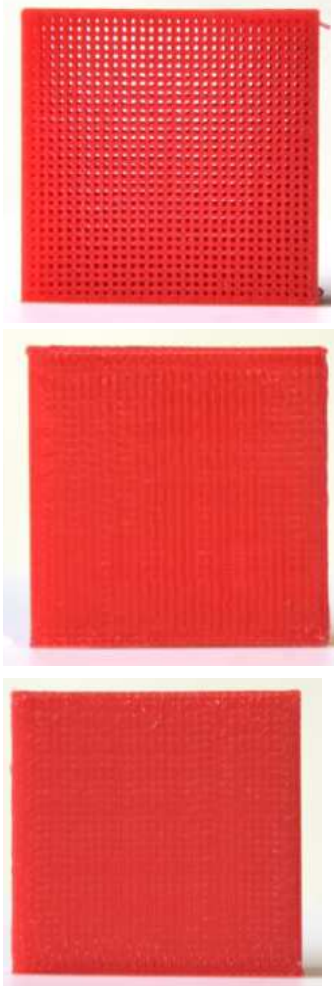
Hexagonal
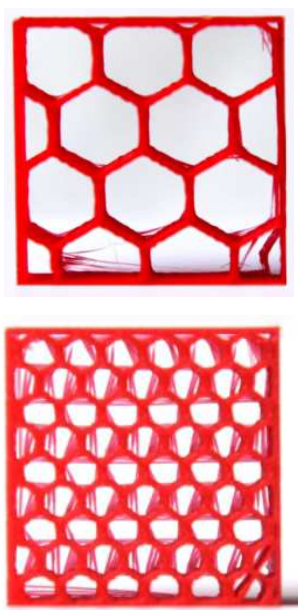

Moroccan star
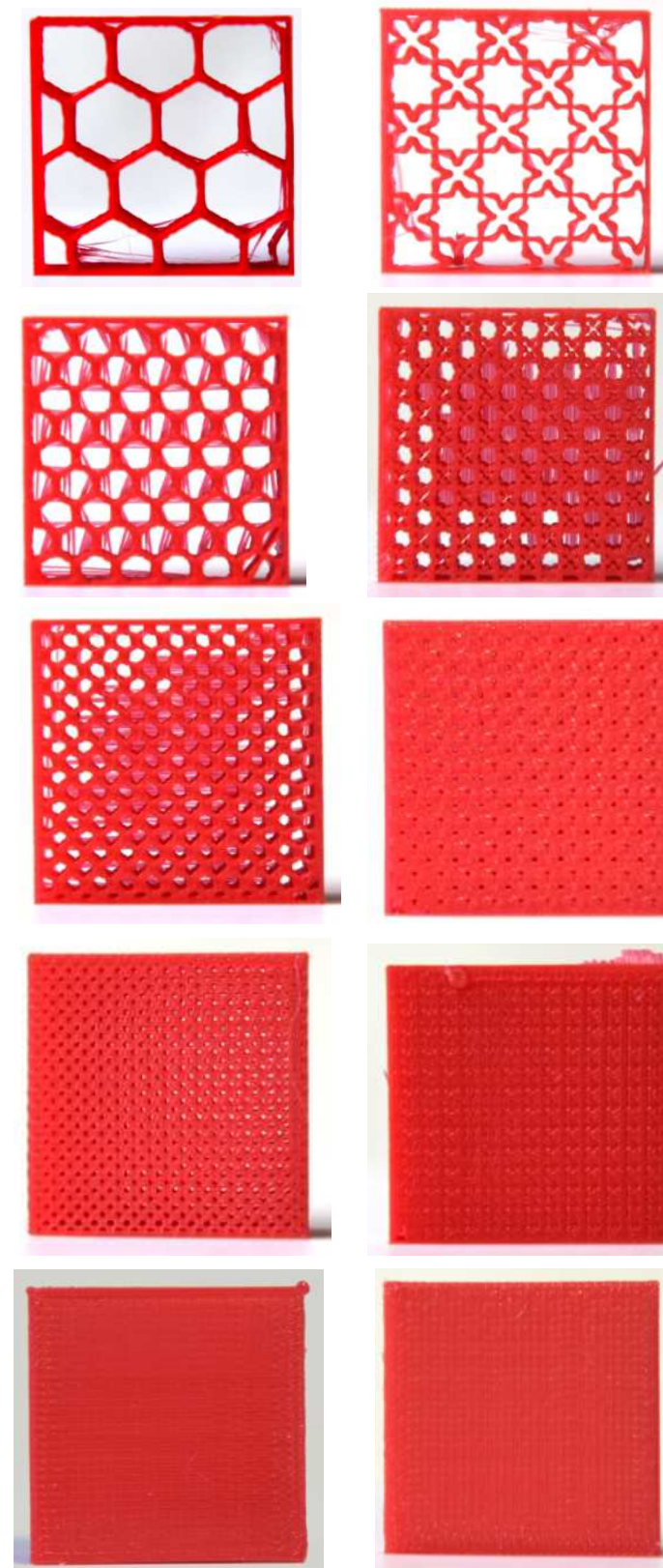

Diamond
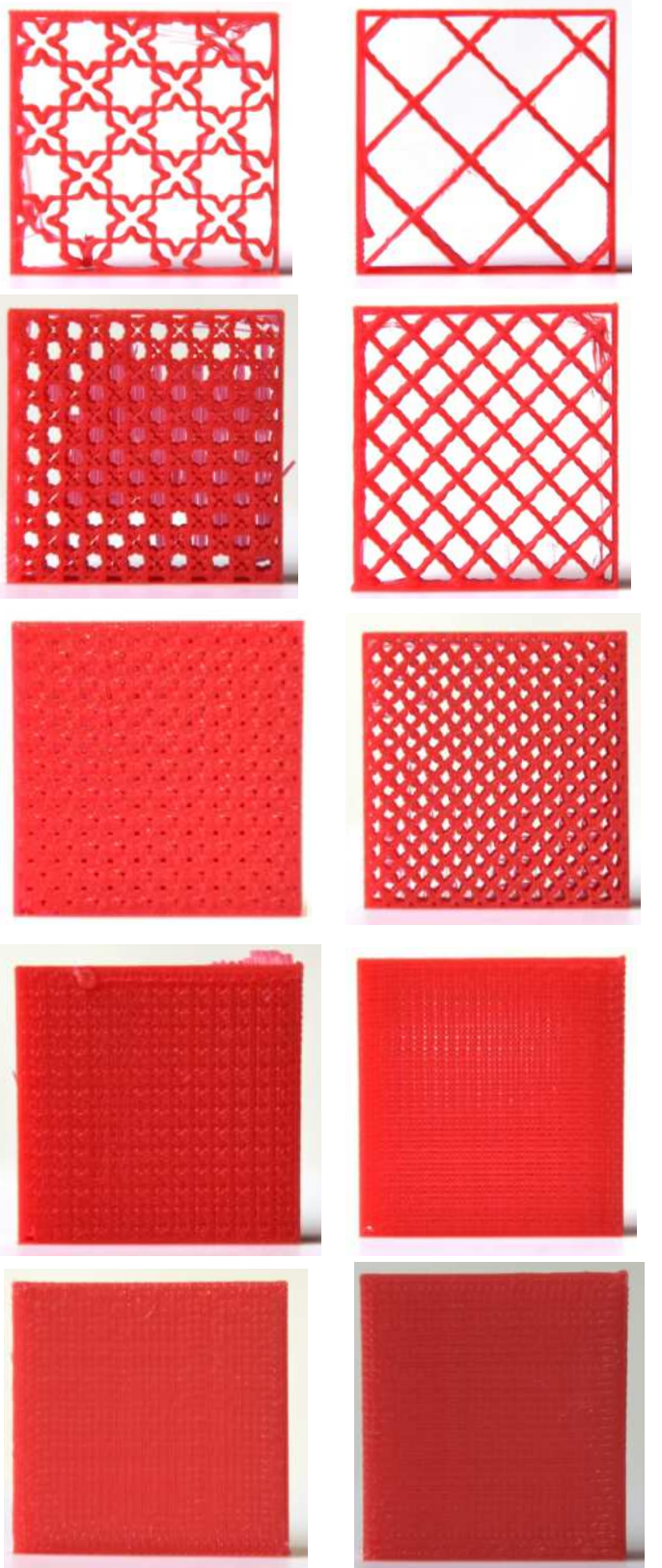


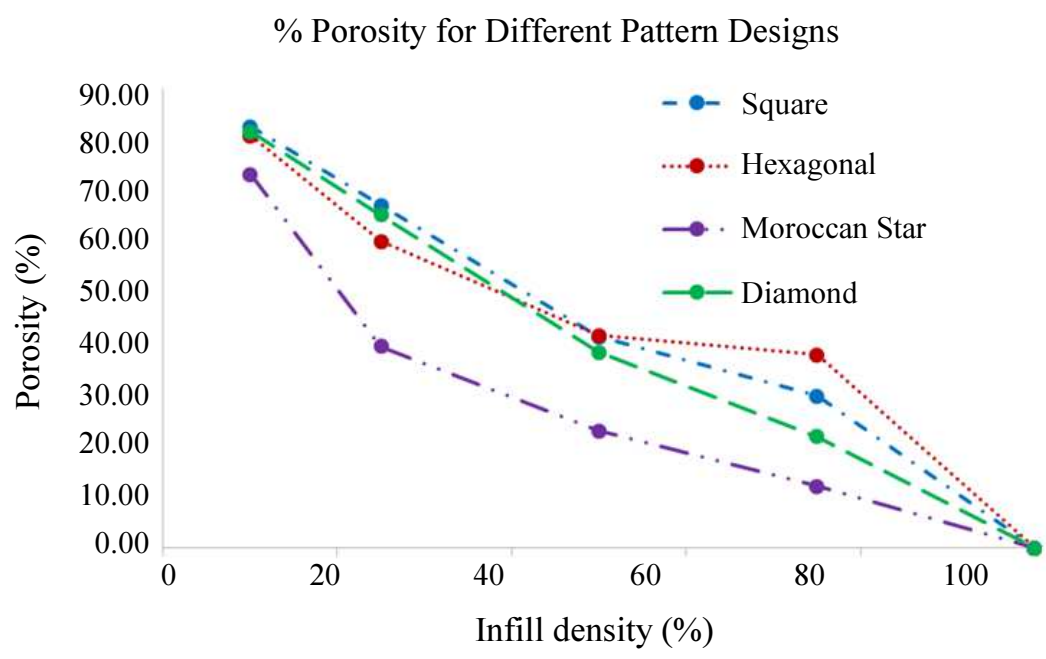

Fig. 4: Porosity of different pattern designs and in-fill densities

Table 2: Weigh measurement for the specimens

\begin{tabular}{lllll}
\hline Infill & & & Moroccan \\
Density (\%) & Linear & Hexagonal & Star & Diamond \\
\hline $10 \%$ & 0.90 & 0.99 & 1.39 & 0.96 \\
$25 \%$ & 1.71 & 2.08 & 3.15 & 1.81 \\
$50 \%$ & 3.06 & 3.04 & 4.02 & 3.22 \\
$75 \%$ & 3.66 & 3.24 & 4.59 & 4.08 \\
$100 \%$ & 5.22 & 5.09 & 5.06 & 5.17 \\
\hline
\end{tabular}

Table 3: Volume measurement for the specimens

\begin{tabular}{lllll}
\hline Infill & & & \multicolumn{2}{l}{ Moroccan } \\
Density (\%) & Linear & Hexagonal & Star & Diamond \\
\hline 10 & 0.74 & 0.82 & 1.14 & 0.79 \\
25 & 1.40 & 1.71 & 2.59 & 1.48 \\
50 & 2.50 & 2.49 & 3.29 & 2.64 \\
75 & 3.00 & 2.66 & 3.76 & 3.34 \\
100 & 4.26 & 4.26 & 4.26 & 4.26 \\
\hline
\end{tabular}

The square pattern had the highest porosity for all infill densities followed by diamond and hexagonal patterns. However, at higher in-fill densities (above $50 \%$ ) the hexagonal pattern had a higher porosity as compared to all pattern designs. This finding is consistent with the fact that a honeycomb design offers higher strength to weight ratio over other in-fill unit designs (Yamashita and Gotoh, 2005).

It is important to note that different pattern designs offer conducive growth platform for the differentiation and proliferation of cells (Huang and Ingber, 1999). Thus, by implementing unit cell designs in conformance with a specific cell lineage, can promote cellular functions such as cell adhesion, orientation, migration and cytoskeletal organization. In addition, the ability to control the porosity of substrate has a significant influence on cell in-growth and impregnation of growth factors and nutrients. A variety of cell types such as fibroblasts, osteoblast, nerve cells and mesenchymal stem cells have shown an affinity towards grooved substrates (Charest et al., 2004; Curtis et al., 2004; Recknor et al., 2004).

In tissue engineering applications it is important to balance the porosity along with the load-bearing capacity of implants. This research enables the selection of optimal pattern designs along with the appropriate porosity for tissue engineering applications.

Table 5 shows the standard tensile test specimens fabricated with different in-fill densities $(10 \%, 50 \%$, and $100 \%$ ) for a square pattern design. The specimens were built in orthogonal orientations (flat, on-edge, and upright). As can be seen, the on-edge orientation has the densest cross-section as compared to other orientations. The orientation also has an impact on the material strength based on the directional properties of the specimens. The effect of different pattern designs and infill density on the strength of the material was evaluated using finite element modeling.

\section{Computational Modeling of 3D Printed Scaffolds}

Finite Element Analysis (FEA) was employed to evaluate the effect of unit-cell design and porosity on the mechanical strength of the specimens. Computational models were run for both tensile and compression testing as per ASTM standards. Figure 5 shows the stress and displacement profiles for both ASTM specimens subjected to a load of $100 \mathrm{~N}$. The von Mises yield criteria based on the maximum distortion energy criterion was used to evaluate the specimens. A maximum stress of $2.836 \mathrm{MPa}$ was recorded for the compressive test and $6.33 \mathrm{MPa}$ for the tensile test. Similarly, the compression specimen had lower displacement $(8.865 \mathrm{e}-2 \mathrm{~mm})$ as compared to the tensile specimen $(1.765 \mathrm{e}-1 \mathrm{~mm})$. Our results are consistent with findings in literature wherein the strength of the human femoral bone is superior in compression loading mode versus tension (Havaldar et al., 2014). 
The tension and compression specimens were modeled with circle, square, diamond and hexagonal unit-cells. The porosity of the unit-cell structures was varied at $10 \%, 25 \%, 50 \%$ and $70 \%$, respectively. The solid specimens in both the loading modes had the lowest stress and displacement values. The diamond pattern had the highest stress and displacement values followed by the circle and square patterns, respectively. The hexagonal unit-cell design had the lowest displacement and stress values of all the pattern designs.

Table 5: Standard test specimens with in-fill density in orthogonal directions

\begin{tabular}{lll}
\hline & Infill Density & \\
Orientation & $10 \%$ & $50 \%$ \\
\hline
\end{tabular}

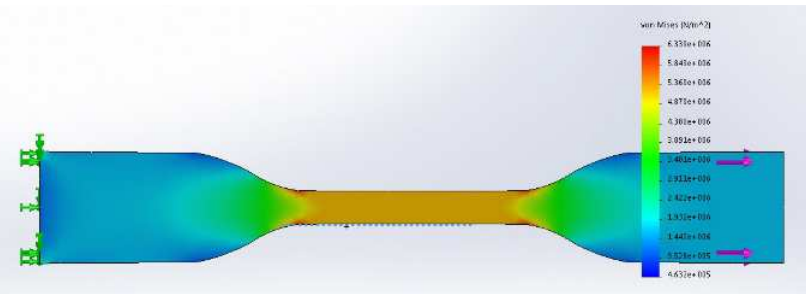

(a)

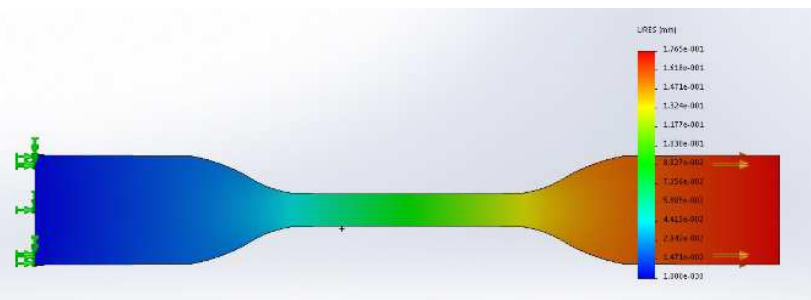

(b)

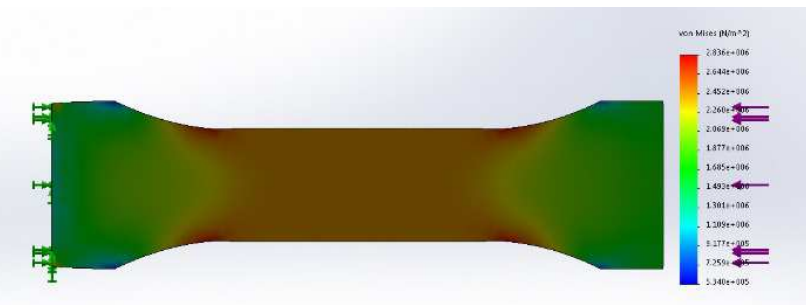

(c)

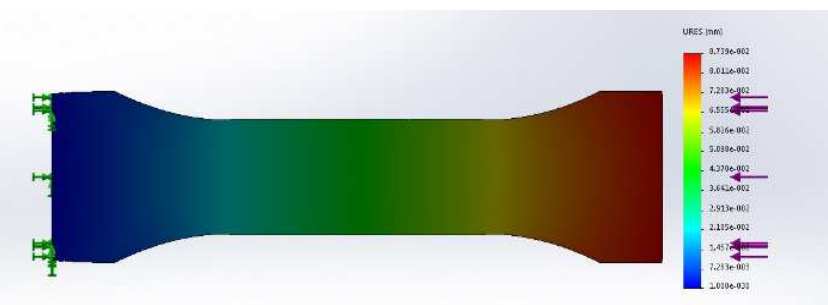

(d)

Fig. 5: Tensile testing specimen (a) stress (b) displacemen. Compression testing specimen (c) stress and (d) displacement 
The specimens undergoing compressive tests (Figure 6) had lower stress and displacement values as compared to the tensile tests (Figure 7). This behavior was consistent across different porosity values and unit-cell patterns. The specimens with compressive loading had approximately $50 \%$ displacement values as compared to tensile specimens. The strength of the specimens decreased with an increase in the porosity. There was a drastic increase in the stress and displacement values for specimens with $70 \%$ porosity as the von Mises stress approach the yield strength of the material.

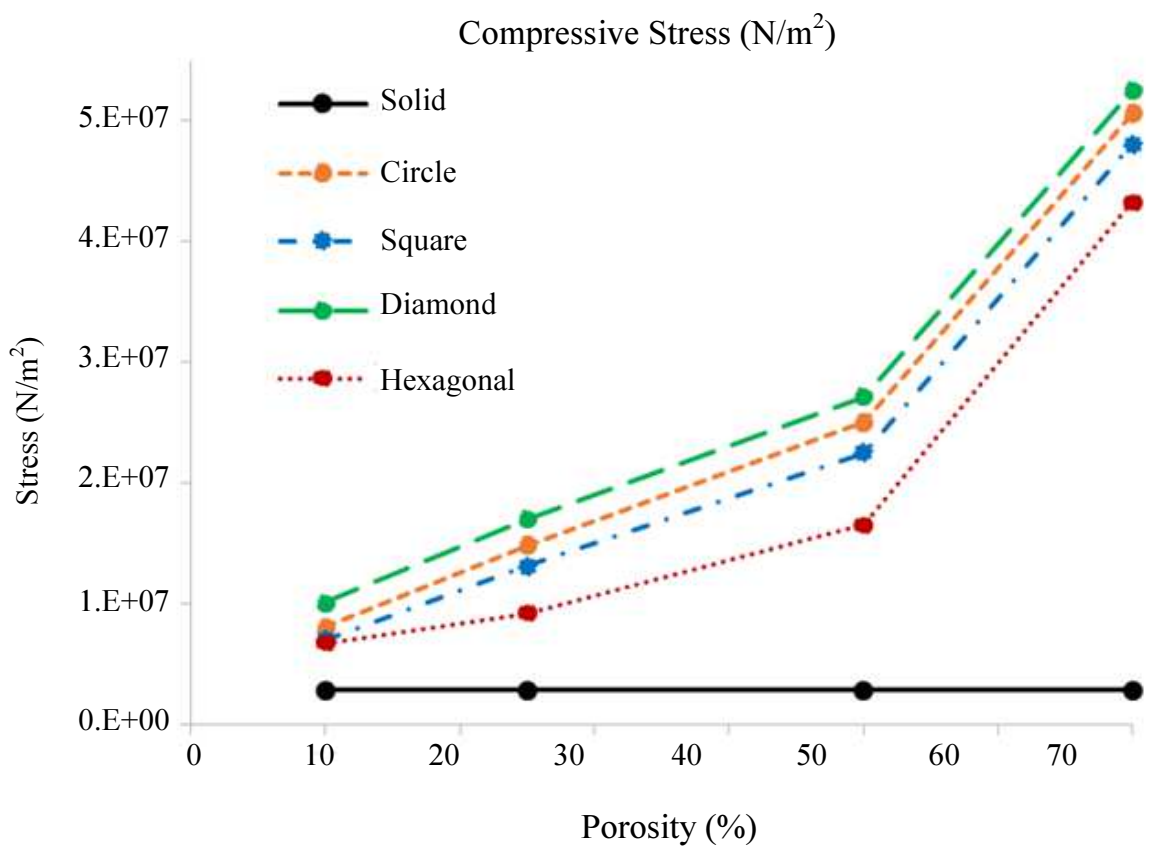

(a)

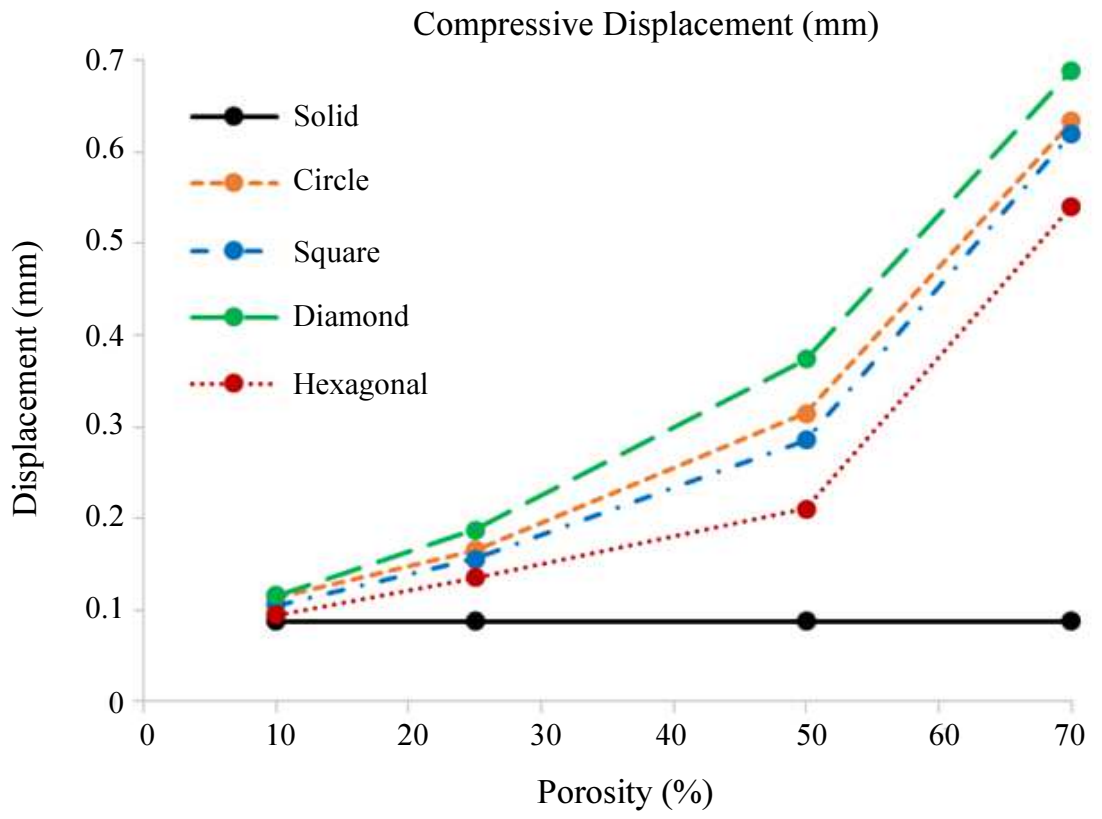

(b)

Fig. 6: Compressive test (a) Stress (b) Displacement 


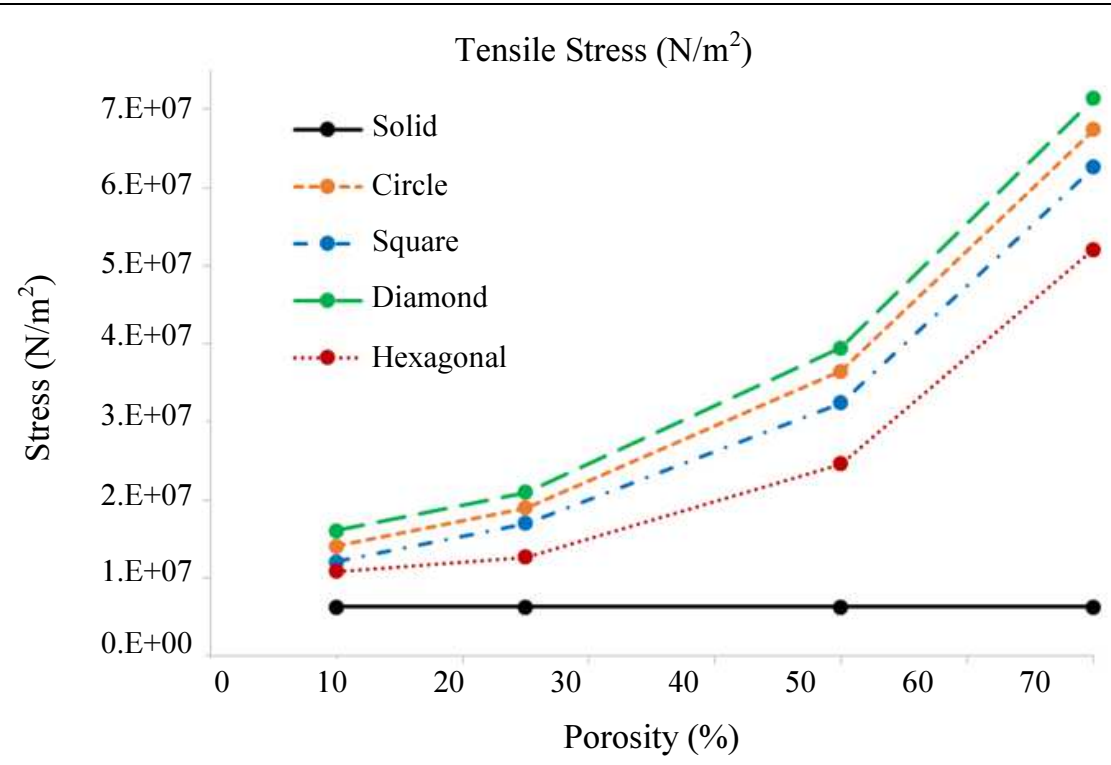

(a)

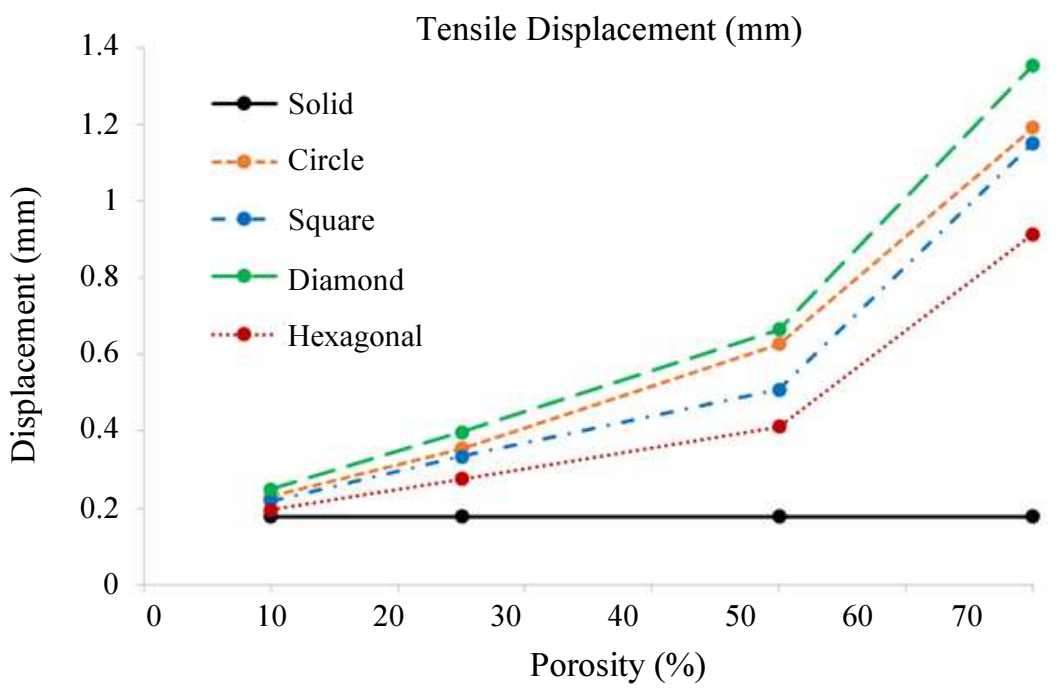

(b)

Fig. 7: Tensile test (a) Stress and (b) Displacement

\section{Conclusion}

This paper investigates 3D printed scaffold structures using experimental analysis and computational models. The fused deposition modeling technique was used to fabricate specimens in ABS material for different unitcell pattern designs. The in-fill density of patterns was varied in $25 \%$ increments for square, diamond, Moroccan star and hexagonal unit-cells. Phase one of experimentation was focused on the control the porosity of scaffold structures. The Moroccan star pattern had the lowest porosity for all in-fill densities whereas the square pattern had the highest porosity. The hexagonal pattern had higher porosity at $75 \%$ in-fill density. ASTM standard tensile testing specimens showed variable porosity based on the orientation of the square in-fill pattern. Thus, by controlling the unit-cell pattern, in-fill density and orientation an optimal porosity can be attained for specific tissue engineering applications.

Phase two of this research included the computational modeling of ASTM tensile and compressive specimens. Finite element analysis was implemented to evaluate the von Mises stress and displacement for different unit-cell patterns with varying porosities. A decrease in mechanical strength was observed with increasing porosity of the specimens. Specimens loaded in the 
compression mode had lower stress and displacement values as compared to the tensile tests. The hexagonal unit-cell design had the highest strength of all the patterns for all in-fill densities in both compression and tension tests. Thus, incorporating a specific unitcell design with optimal porosity can balance cellular functions with the load-bearing capacity of the implant. This research lays the basis for developing custom scaffolds for stem-cell based tissue engineering applications.

\section{Funding Information}

This research was funded by US National Science Foundation (NSF CMMI: Award 1663128).

\section{Author's Contributions}

Ablah Aljohani and Salil Desai: Performed simulation work and conducted analysis of the research. All authors contributed to the writing of the manuscript.

\section{Ethics}

The authors declare that there are no ethical issues that could arise after the publication of this study.

\section{References}

Banks, J., 2013. Adding value in additive manufacturing: researchers in the United Kingdom and Europe look to 3D printing for customization. IEEE Pulse, 4: 22-26. DOI: 10.1109/MPUL.2013.2279617

Bencherif, S.A., T.M. Braschler and P. Renaud, 2013. Advances in the design of macroporous polymer scaffolds for potential applications in dentistry. J. Periodontal Implant Sci., 43: 251-261. DOI: 10.5051/jpis.2013.43.6.251

Bose, S., M. Roy and A. Bandyopadhyay, 2012. Recent advances in bone tissue engineering scaffolds. Trends Biotechnol., 30: 546-554. DOI: 10.1016/j.tibtech.2012.07.005

Charest, J.L., L.E. Bryant, A.J. Garcia and W.P. King, 2004. Hot embossing for micropatterned cell substrates. Biomaterials, 25: 4767-4775. DOI: $10.1016 /$ j.biomaterials.2003.12.011

Curtis, A., N. Gadegaard, M. Dalby, M. Riehle and C. Wilkinson, 2004. Cells react to nanoscale order and symmetry in their surroundings. IEEE Trans. Nanobiosci., 3: 61-65. DOI: $10.1109 /$ TNB.2004.824276

Dadsetan, M., T.E. Hefferan, J.P. Szatkowski, P.K. Mishra and S.I. Macura, 2008. Effect of hydrogel porosity on marrow stromal cell phenotypic expression. Biomaterials, 29: 2193-2202.

DOI: 10.1016/j.biomaterials.2008.01.006
Desai, S. and B. Harrison, 2010. Direct-writing of biomedia for drug delivery and tissue regeneration. Printed Biomaterials. DOI: $10.1007 / 978-1-4419-1395-15$

Desai, S., J. Perkins, B.S. Harrison and J. Sankar, 2010. Understanding release kinetics of biopolymer drug delivery microcapsules for biomedical applications. Materials Sci. Eng., 168: 127-131. DOI: 10.1016/j.mseb.2009.11.006

Dhandayuthapani, B., Y. Yoshida, T. Maekawa and D.S. Kumar, 2011. Polymeric scaffolds in tissue engineering application: A review. Int. J. Polymer Sci., 2011: 1-19. DOI: 10.1155/2011/290602

Gerhardt, L.C. and A.R. Boccaccini, 2010. Bioactive glass and glass-ceramic scaffolds for bone tissue engineering. Materials, 3: 3867.

DOI: $10.3390 / \mathrm{ma3} 073867$

Havaldar, R., S. Pilli and B. Putti, 2014. Insights into the effects of tensile and compressive loadings on human femur bone. Advanced Biomedical Res. DOI: $10.4103 / 2277-9175.129375$

Huang, S. and D.E. Ingber, 1999. The structural and mechanical complexity of cell-growth control. Nature Cell Biology, 1: E131. DOI: 10.1038/13043

Hutmacher, D.W., 2001. Scaffold design and fabrication technologies for engineering tissues-state of the art and future perspectives. J. Biomaterials Sci., 12: 107-124. DOI: $10.1163 / 156856201744489$

Karageorgiou, V. and D. Kaplan, 2005. Porosity of 3D biomaterial scaffolds and osteogenesis. Biomaterials, 26: 5474-5491. DOI: $10.1016 /$ j.biomaterials.2005.02.002

Klein, G.T., Y. Lu and M.Y. Wang, 2013. 3D printing and neurosurgery - ready for prime time? World Neurosurgery, 80: 233-235.

DOI: 10.1016/j.wneu.2013.07.009

Liu, S., T. Schackel, N. Weidner and R. Puttagunta, 2017. Biomaterial-supported cell transplantation treatments for spinal cord injury: Challenges and perspectives. Frontiers Cellular Neurosci., 11: 430. DOI: 10.3389/fncel.2017.00430

Luo, Y., D. Zhai, Z. Huan, H. Zhu and L. Xia, 2015. Three-dimensional printing of hollow-struts-packed bioceramic scaffolds for bone regeneration. ACS Applied Materials Interfaces, 7: 24377-24383. DOI: 10.1021/acsami.5b08911

Murphy, C.M., M.G. Haugh and F.J. O'brien, 2010. The effect of mean pore size on cell attachment, proliferation and migration in collagenglycosaminoglycan scaffolds for bone tissue engineering. Biomaterials, 31: 461-466. DOI: 10.1016/j.biomaterials.2009.09.063

Pan, Z., P. Duan, X. Liu, H. Wang and L. Cao, 2015. Effect of porosities of bilayered porous scaffolds on spontaneous osteochondral repair in cartilage tissue engineering. Regenerative Biomaterials, 2: 9-19. DOI: $10.1093 / \mathrm{rb} / \mathrm{rbv} 001$ 
Parupelli, S.K., 2016. Understanding hybrid additive manufacturing of functional devices.

Perkins, J., H. Yi, S.H. Ye, W. Wagner and S. Desai, 2014. Direct write manufacturing of controlled release coatings for drug eluting cardiovascular stents. J. Biomedical Res. Part A. DOI: $10.1002 / \mathrm{jbm} . \mathrm{a} .35105$

Perkins, J., Z. Xu, C. Smith, A. Roy and P.N. Kumta, 2015. Direct writing of polymeric coatings on magnesium alloy for tracheal stent applications. Annals Biom. Eng., 43: 1158-1165. DOI: $10.1007 / \mathrm{s} 10439-014-1169-3$

Ramalingam, M., E. Jabbari, S. Ramakrishna and A. Khademhosseini, 2013. Micro and nanotechnologies in engineering stem cells and tissues, John Wiley \& Sons.

Recknor, J.B., J.C. Recknor, D.S. Sakaguchi and S.K. Mallapragada, 2004. Oriented astroglial cell growth on micropatterned polystyrene substrates. Biomaterials, 25: 2753-2767.

DOI: 10.1016/j.biomaterials.2003.11.045
Schubert, C., M.C. Van Langeveld and L.A. Donoso, 2014. Innovations in 3D printing: A 3D overview from optics to organs. Brit. J. Ophthalmology, 98: 159-161. DOI: 10.1136/bjophthalmol-2013-304446

Ursan, I.D., L. Chiu and A. Pierce, 2013. Threedimensional drug printing: A structured review. J. Am. Pharmacists Association, 53: 136-144. DOI: 10.1331/JAPhA.2013.12217

Yamashita, M. and M. Gotoh, 2005. Impact behavior of honeycomb structures with various cell specifications-numerical simulation and experiment. Int. J. Impact Eng., 32: 618-630. DOI: 10.1016/j.ijimpeng.2004.09.001 